\title{
Design and implementation of the NUV/optical widefield Star Formation Camera for the Theia observatory
}

Paul A. Scowen*a, Rolf H. Jansen ${ }^{\mathrm{a}}$, Matthew N. Beasley ${ }^{\mathrm{b}}$, Daniella Calzetti ${ }^{\mathrm{c}}$, Steve Desch", Alex W. Fullerton ${ }^{\mathrm{d}}$, John S. Gallagher III ${ }^{\mathrm{e}}$, P. Douglas Lisman ${ }^{\mathrm{f}}$, Steven A. Macenka ${ }^{\mathrm{f}}$, Sangeeta Malhotra ${ }^{\mathrm{a}}$, Mark J. McCaughrean ${ }^{\mathrm{g}}$, Shouleh Nikzad ${ }^{\mathrm{f}}$, Robert W. O'Connell ${ }^{\mathrm{h}}$, Sally Oey ${ }^{i}$, Deborah L. Padgett ${ }^{j}$, James E. Rhoads ${ }^{a}$, Aki Roberge ${ }^{k}$, Oswald H. W. Siegmund ${ }^{1}$, Stuart B. Shaklan ${ }^{\mathrm{f}}$, Nathan Smith ${ }^{\mathrm{m}}$, Daniel Stern ${ }^{\mathrm{f}}$, Jason Tumlinson ${ }^{\mathrm{d}}$, Rogier A. Windhorst ${ }^{\mathrm{a}}$, Robert A. Woodruff ${ }^{n}$

${ }^{a}$ School of Earth \& Space Exploration, Arizona State University, PO Box 871404, Tempe, AZ USA $85287-1404$

b Astrophysics Research Laboratory, University of Colorado at Boulder - CASA, Boulder, CO USA 80309-0593;

${ }^{c}$ Dept. of Astronomy, University of Massachusetts, Amherst, MA USA 01003

${ }^{\mathrm{d}}$ Space Telescope Science Institute, 3700 San Martin Drive, Baltimore, MD USA 21218

${ }^{e}$ Dept. of Astronomy, University of Wisconsin - Madison, 475 N. Charter St., Madison, WI USA 53706-1582

${ }^{\mathrm{f} J e t}$ Propulsion Laboratory, 4800 Oak Grove Dr., Pasadena, CA USA 91109

${ }^{\mathrm{g}}$ School of Physics, University of Exeter, Stocker Road, Exeter UK EX4 4QL

${ }^{\mathrm{h}}$ Dept. of Astronomy, University of Virginia, P.O. Box 400325, Charlottesville, VA USA 229044325

i Dept. of Astronomy, University of Michigan, 830 Dennison Bldg., Ann Arbor, MI USA 481091042

${ }^{\mathrm{j}}$ Spitzer Science Center, Caltech, Pasadena, CA USA 91125

${ }^{\mathrm{k}}$ NASA GSFC, Code 667, Greenbelt, MD USA 20771

${ }^{1}$ Space Science Lab., University of California - Berkeley, Berkeley, CA USA 94720

${ }^{\mathrm{m}}$ Astronomy Dept., 601 Campbell Hall, University of California at Berkeley, Berkeley, CA USA 94720-3411

${ }^{\mathrm{n}}$ Lockheed Martin Space Systems Co., 6304 Spine Rd., Boulder, CO USA 80301-3674

\begin{abstract}
The Star Formation Camera (SFC) is a wide-field $\left(\sim 19^{\prime} \times 15^{\prime},>280 \operatorname{arcmin}^{2}\right)$, high-resolution (18 mas pixels) UV/optical dichroic camera designed for the Theia 4-m space-borne space telescope concept. SFC will deliver diffraction-limited images at $\lambda>300 \mathrm{~nm}$ in both a blue $(190-517 \mathrm{~nm})$ and a red $(517-1075 \mathrm{~nm})$ channel simultaneously. The goal is to conduct a comprehensive and systematic study of the astrophysical processes and environments relevant for the births and life cycles of stars and their planetary systems, and to investigate the range of environments, feedback mechanisms, and other factors that most affect the outcome of star and planet formation.
\end{abstract}

Keywords: NUV, optical, large format focal planes, silicon, CCD, dichroic, filters, space-based, anastigmat

*paul.scowen@asu.edu; phone 1480 965-0938; fax 1480 965-8102; sese.asu.edu; sfc.asu.edu

\section{INTRODUCTION}

Over the past 25 years, the Hubble Space Telescope has revolutionized our view of the universe, excited and engaged the general public with its compelling images, and has been a workhorse for astrophysics. As part of a larger team, we have designed and studied a mission called THEIA ${ }^{1}$, Telescope for Habitable Exoplanets and Interstellar/Intergalactic

Space Telescopes and Instrumentation 2010: Optical, Infrared, and Millimeter Wave, edited by Jacobus M. Oschmann Jr., Mark C. Clampin, Howard A. MacEwen, Proc. of SPIE Vol. 7731,77314 Y · C 2010 SPIE · CCC code: 0277-786X/10/\$18 · doi: 10.1117/12.857475 
Astronomy, a flagship 4-meter on-axis optical/UV telescope as a worthy successor to HST and companion to the James Webb Space Telescope (JWST). With a wide field imager, an ultraviolet spectrograph, a planet imager/spectrograph and a companion occulter, THEIA is capable of addressing many of the most important questions in astronomy: Are we alone? Are there other habitable planets? How frequently do solar systems form and survive? How do stars and galaxies form and evolve? How is dark matter distributed in galaxies and in the filaments? Where are most of the atoms in the universe? How were the heavy elements necessary for life created and distributed through cosmic time?

The THEIA Observatory, schematically shown in Figure 1, is an on-axis three-mirror anastigmat (TMA) telescope with a 4-meter $\mathrm{Al} / \mathrm{MgF}_{2}$-coated primary, an $\mathrm{Al} / \mathrm{LiF}$-coated secondary and three main instruments: Star Formation Camera ${ }^{2}$ (SFC), a dual-channel wide field UV/optical imager covering $19^{\prime} \times 15^{\prime}$ on the sky with 18 mas pixels; UltraViolet Spectrograph $^{3}$ (UVS), a multi-purpose spectrometer optimized for high sensitivity observations of faint astronomical sources at spectral resolutions, $\lambda / \Delta \lambda$, of 30,000 to 100,000 in the $100-300 \mathrm{~nm}$ wavelength range; and eXtrasolar Planet Characterizer (XPC), which consists of three narrow-field cameras $(250-400 \mathrm{~nm} ; 400-700 \mathrm{~nm} ; 700-1000 \mathrm{~nm})$ and two $\mathrm{R} / 70$ integral field spectrographs (IFS).

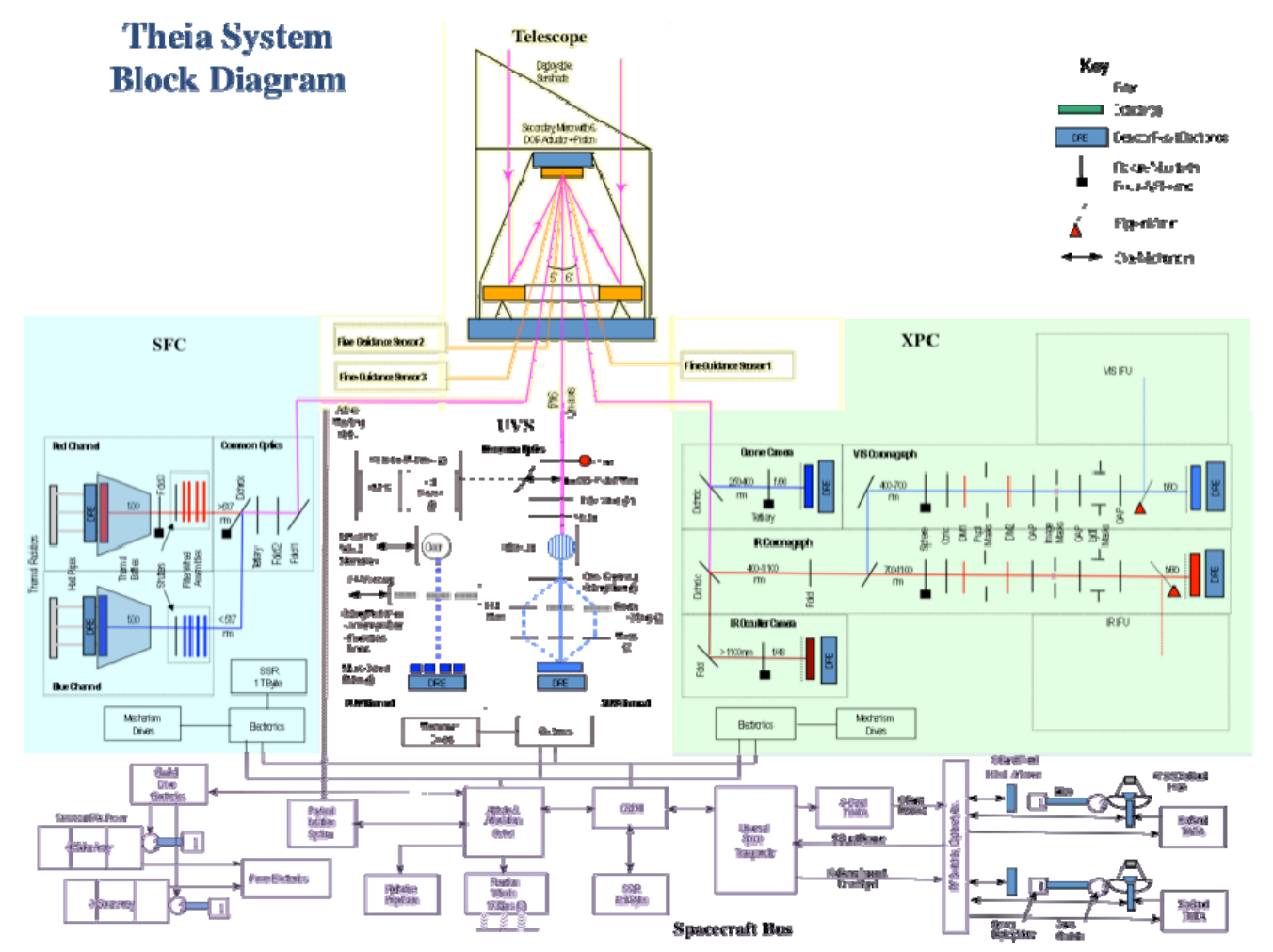

Figure 1. Block diagram of the Theia Observatory design. The TMA design feeds three different instrument chains that pick off from different parts of the focal plane. Each uses a different tertiary to deliver a different beam speed, with the UVS having no tertiary to minimize UV losses due to reflection.

\section{INSTRUMENT CONCEPT}

\subsection{Overview}

The SFC instrument, shown in Figure 2, is a wide-field survey imager with an unprecedented combination of FOV $\left(19^{\prime} \times 15^{\prime}\right)$ and resolution (18 mas). Final resolution is diffraction limited at $300 \mathrm{~nm}$, but camera size is reduced by undersampling at the diffraction limit. Critical sampling is reconstructed on the ground from multiple images with dithered pointing, using fast steering mirrors (FSMs) within the instrument. The camera has been designed to take advantage of the wide, well-corrected field delivered by the observatory TMA design. 
A dichroic splits the beam at $517 \mathrm{~nm}$ into simultaneously operated red (517-1075nm) and blue (190-517nm) channels, for efficient observing. Each channel includes a tailored set of band-pass filters and a shutter, as well as its own FSM and focus mechanism. The combination of FSM and spacecraft stability will deliver a jitter spot less than 1/4 pixel in size.

SFC is a standalone instrument designed to be operated during periods when the XPC instrument is not observing, but can also be operated in parallel to obtain deep cosmological images while dwelling on planetary candidates.

The camera employs the same foundry CCD chips on both channels, post processed differently to optimize each channel for the best sensitivity possible, to mitigate both cost and risk associated with populating such large focal planes.

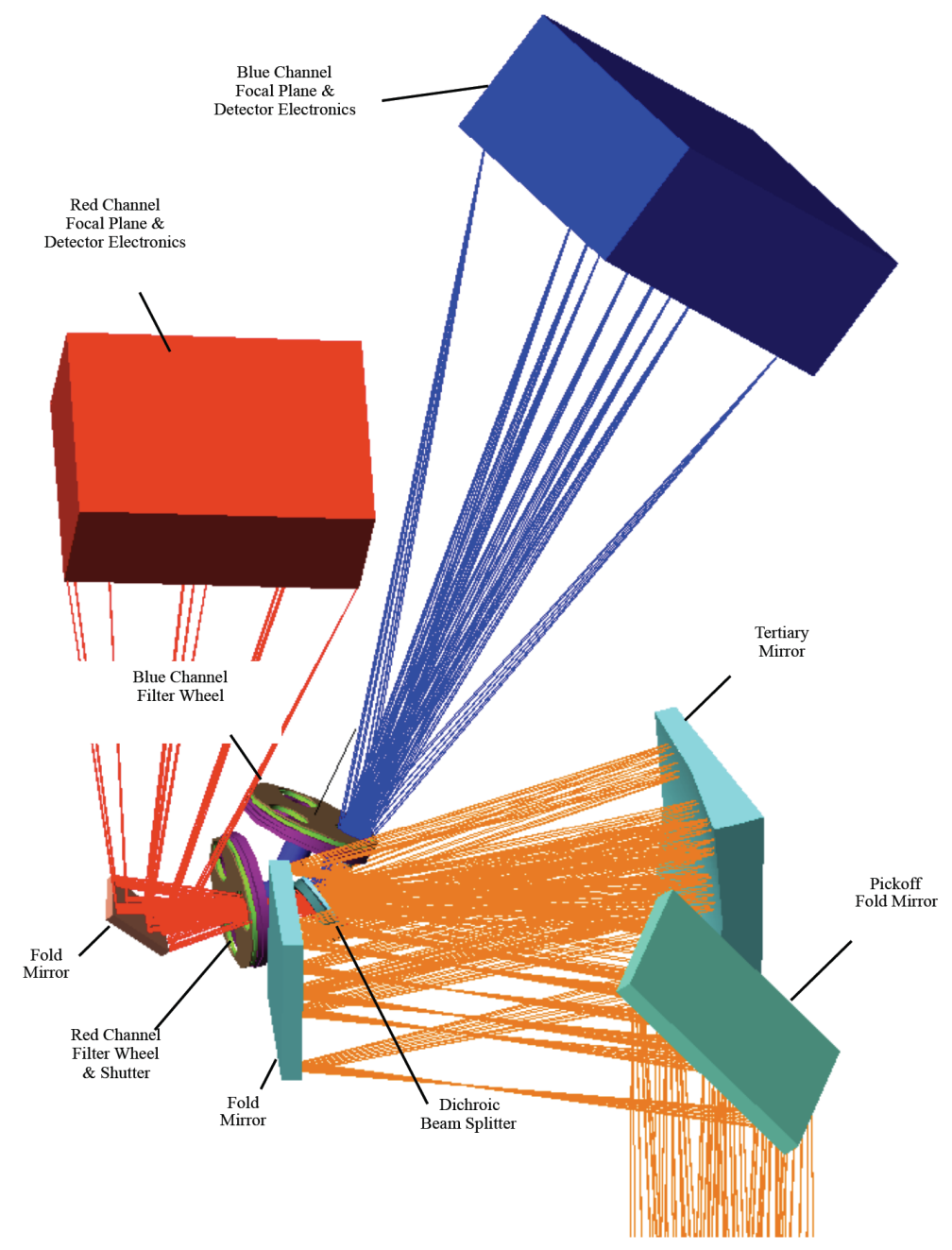

Figure 2. Primary design components of the Star Formation Camera. The beam from the telescope is picked off and fed by 2 fold mirrors to the tertiary mirror of the 3-mirror anastigmat design. The beam is then split by a dichroic element and sent to two parallel optical channels, each with its own filter wheel and focal plane.

\subsection{Focal Plane Array Design}

We have designed SFC to use Si CCD chips that have been optimized and custom processed to maximize their response across the full Si-response passband $(190-1075 \mathrm{~nm})$ - our baseline detectors are the SNAP-like LBNL $3.5 \mathrm{k} \times 3.5 \mathrm{k}$ $10.5 \mu \mathrm{m}$ pixel chips, but post-processed using JPL's Delta Doping technique and AR coated to make each chip as sensitive as possible.

The design deliberately uses the same foundry chip but processed differently to provide optimal chips for each of two observational channels - one in the blue (190-517nm) and one in the red (517-1075nm) - to drive down the cost of populating the FPAs. 
The optical design delivers 18 mas pixels, which is the Rayleigh criterion for a $4 \mathrm{~m}$ mirror at $300 \mathrm{~nm}$, which is halfNyquist sampling. As a result, operation of the camera requires the use of a dithering strategy (see below) to recover the diffraction limit at the shortest wavelengths, but is also required at longer wavelengths to recover spatial coverage.

The camera has been designed to have as wide a field as possible to open up a unique capability of wide field AND high resolution imaging - the current design has a 4:3 aspect delivering a $19^{\prime} \times 15^{\prime}$ field of view - this corresponds to arrays of $18 \times 15$ CCD chips for a total of 270 chips per FPA. This solution provides the right balance between field size and resolution. It is not a perfect solution but it is the most practical.

Each image taken with SFC, with the 2 channels in parallel, produces twin 6.3 GB images. We require on-board storage for 2 days of data, which could be as much as 12 TB per day, uncompressed. We employ lossless compression to reduce the daily data load to $6 \mathrm{~TB}$ - an amount of data that can be downloaded in 8-10 hours every 24-hr period using Ka-band. We have specified twice this value in storage capacity in the design to allow for a loss of one downlink opportunity without impact on the observing sequence of the camera.

The camera FPAs will be operated at a temp of $175 \mathrm{~K}$ to deliver a dark current of less than $10 \mathrm{e}-/ \mathrm{pix} / \mathrm{hr}$ with a read noise of less than $3 \mathrm{e}-$, at a gain of $2 \mathrm{e}-/ \mathrm{ADU}$ with a full-well capacity of 130,000 e-.

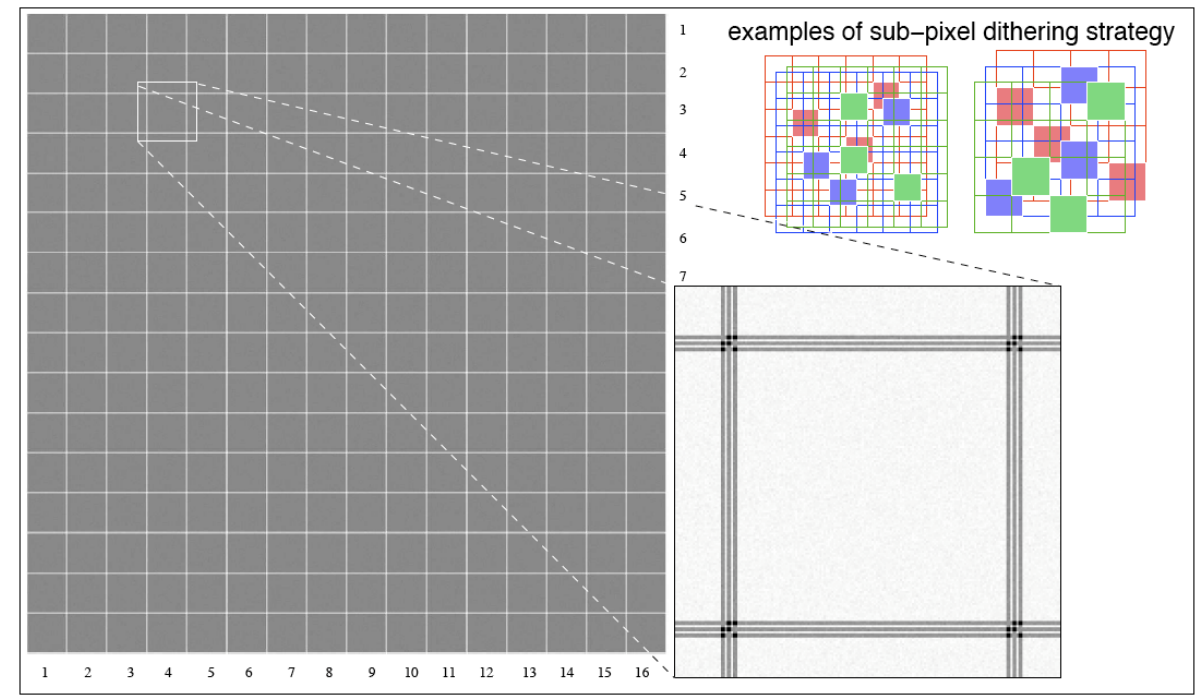

Figure 3. SFC survey tile, a FPA mosaic or stack of 9 individual exposures at 3 different pattern pointings (with 3 sub-pixel dither/CRSPLIT exposures) that is our assumed base-line survey strategy to provide full areal coverage, reject detector defects and cosmic rays, and reconstruct the PSF delivered by the $4.0 \mathrm{~m}$ Theia OTA. With this strategy, $92.2 \%$ of the area will have the full exposure time, $7.7 \%$ of the area will have $2 / 3$ of the full exposure time, and only $0.1 \%$ will have $1 / 3$ of that time and no means to reject detector defects (but will still be free of cosmic ray induced signal thanks to the sub-pixel dithers).

\subsection{Observing with the SFC}

The SFC has been deliberately designed to be a powerful survey instrument for observation and measurement of star formation both local and extragalactic. Some of its features have been driven by this intent.

The camera has an extensive science-driven complement of both broad- and narrow-band filters (see Table 1). The combination of the large $4 \mathrm{~m}$ aperture with the 6 optical bounces (driven by design and packaging issues) yields a high throughput system that can in a 2000 second exposure reach down to around 29th magnitude in the broader filters, and down to surface brightnesses of $10^{-16} \mathrm{ergs} / \mathrm{cm}^{2} / \mathrm{s} / \operatorname{arcsec}^{2}$.

The camera chips cannot be butted causing inter-chip gaps of order 35 pixels in size - removal of these as well as recovery of the diffraction limit at $300 \mathrm{~nm}$ requires each pointing to be comprised of 9 exposures: 3 microdithers at the 1/4 pixel level $\times 3$ macrodithers to fill the omitted coverage (see Figure 3 ). 
Table 1: Instrument Properties for the Star Formation Camera

\begin{tabular}{|c|c|}
\hline Operating Wavelengths & 190-1075nm (Si sensitivity passband) \\
\hline Observing Channels & Blue (190-517nm); Red (517-1075nm) \\
\hline Broad-band Filters & $\begin{array}{l}\text { F241X, F262W, F278XX, F312X, F330W, F385X, F432W, } \\
\text { F467X, F578X, F612W, F707X, F775W, F867X, F885W }\end{array}$ \\
\hline Medium-band Filters & F212M, F547M, F920M, F948M, F980M, F990M, F1020M \\
\hline Narrow-band Filters & $\begin{array}{l}\text { F280N, F373N, F486N, F470N, F487N, F502N, F632N, } \\
\text { F656N, F658N, F659N, F674N, F956N }\end{array}$ \\
\hline Grism Filters & G213L, G402M, G745L, G895M \\
\hline Exposure Times & 0.1 up to 2000 seconds \\
\hline Detectors & LBNL "SNAP" 3.5k square CCDs \\
\hline Pixel Size & $10.5 \mu \mathrm{m}=18 \mathrm{mas}$ \\
\hline Field Size & $19^{\prime} \times 15^{\prime}=18 \times 15 \mathrm{CCDs}$ \\
\hline Dark Noise & $<10$ e-/pix/hr \\
\hline Read Noise & $<3 \mathrm{e}-$ \\
\hline Gain & $2 \mathrm{e}-/ \mathrm{ADU}$ \\
\hline Full Well Capacity & $130,000 \mathrm{e}-$ \\
\hline Operational Temperature & $175 \mathrm{~K}$ \\
\hline Pointing Accuracy (w/ FSM) & $<1 / 4$ pixel over 2000 seconds \\
\hline Interchip Gap Size & $\sim 35$ pixels \\
\hline Mass & $645 \mathrm{Kg}$ \\
\hline Power & $424 \mathrm{~W}$ (of which $375 \mathrm{~W}$ for FPAs) \\
\hline Cost (inc. $30 \%$ reserve) & $\$ 390 \mathrm{M}$ \\
\hline Instrumental Lifetime & 5-yr baseline, 10 -yr design \\
\hline Single Field Exposure Image Size & $6.3 \mathrm{~GB} \times 2$ channels \\
\hline On-board capacity & $6 \mathrm{~TB}$ \\
\hline Typical lossless compression & Factor of 2-3.5 \\
\hline Broadband Sensitivity & $\mathrm{m}_{\mathrm{V}} \sim 29$ in 2000 seconds \\
\hline Narrowband Sensitivity & $10^{-16} \mathrm{ergs} / \mathrm{cm}^{2} / \mathrm{s} / \operatorname{arcsec}^{2}$ in 2000 seconds \\
\hline
\end{tabular}

The camera employs a high fidelity dichroic element to allow parallel observations in both the blue and red channels this element also doubles as a FSM for the blue channel - the red channel uses its extra fold mirror as the FSM. Observing guidance for SFC (and other instruments on Theia) is provided by 3 fast CCDs mounted at the Cass-like focal plane behind the primary next to the first pickoff mirror for SFC (see Figure 1) - the signal from these chips is used not only to maintain pointing but to drive the fast steering mirror (FSM) that maintains the jitter histogram at less than the $1 / 4$ pixel specification.

The very small pixels in SFC make it almost impossible to get decent signal from the sky to allow sky-flats - as such we have included an internal CAL channel in the camera design to allow flatfielding using a lightpipe and diffuser comounted in the first filterwheel on each channel. The rotating shutter is comounted in front of each filter assembly and can deliver exposure times ranging from a fraction of a second up to as long as 2000 seconds, and can be used with ND filters in cases of extreme brightness. 


\subsection{Technological Challenges}

The SFC is a very straightforward design and employs many technologies that are of high TRL and have good heritage. However, there are some aspects of the design that will require investment over the next decade to raise their TRL. The manufacture of the SNAP LBNL detectors has been assigned a TRL level of 6 by the SNAP project and 4-5 by BEPAC. The construction of the SFC will require the development of post-processing, testing and characterization of large numbers of CCD chips in a production-line model (see next section). Part of this process is the JPL Delta Doping technology, which has been assigned a TRL of 4 by BEPAC. To store on-board the very large volumes of data, it will be necessary to flight-rate the solid-state storage technology we have baselined for this project.

\section{ASSEMBLY OF LARGE FOCAL PLANE ARRAYS FOR SPACE ${ }^{4}$}

As we move into the next decade in astronomy, it is becoming very apparent that there are a large variety of scientific applications that would benefit dramatically from access to truly large focal plane arrays combined with very small pixel plate scales to provide high angular resolution in tandem to the large areal sample, as represented by SFC. In this section we discuss the technological challenges that have already been studied as part of existing missions and future mission concept studies. We believe this is an area of technological development that is primed for truly innovative advancement with a relatively modest investment over the next 10 years, and the benefit to the scientific community would be considerable.

To support sweeping new science programs, we need to develop the technological capability to construct large focal plane arrays (FPAs) that are flight rated for space in a reliable and straightforward fashion that simultaneously mitigates risk, maximizes yield, and minimizes cost. The scope of this challenge is significant in that it will likely require many years of concerted effort and investment by the entire astronomy community to develop new FPA design, fabrication, and assembly technologies; data handling and storage methodologies; and observational strategies to allow a large scale systems to be built in a routine fashion.

\subsection{Suitable Architecture for Large FPAs}

The design of large focal plane arrays is driven by several factors. One is the set of scientifically-driven requirements for the detectors themselves. Such requirements can include the required passbands for observation, the form factor and pitch of the detectors, the overall size of the array or field of view of the camera, the seam size, the detector clocking rate, the noise characteristics and therefore the operational temperature of the detectors, and considerations for total data storage, compression and transmission. Another factor is that of the electronic architecture that needs to be adopted to minimize the risk associated with integration and testing of the focal plane as it is constructed and prepared for flight.

Development of modular designs for the sensor, its control and readout electronics are recommended, to allow individual detectors to be tested and easily removed as needed, once integrated into the focal plane assembly, with a minimum of impact on any neighboring detectors. Each channel needs to be designed with a dual string layout to allow for failure during the mission, and each chip needs to have a power supply that is as noise free as possible.

\subsection{Populating the Focal Plane - Detector Options}

Two major classes of detectors exist in the UV/optical/NIR spectral range: substrate-removed HgCdTe and silicon imagers. While high quality, large format HgCdTe FPAs are commercially available, the high cost of these FPAs remains a major disincentive to their use in large focal planes that do not require spectral response beyond $1 \mu \mathrm{m}$. The maturity of silicon imagers and their high performance and recent development to extend the spectral range in both long and short wavelength renders silicon imagers as detector of choice for UV/optical/NIR instruments.

Silicon imagers can be divided into those with a high degree of integration (e.g. CMOS imaging arrays with on-chip bias, timing, control and many parallel readouts), CCDs (serial readout, charge transfer to final one or few amplifiers), and hybrid arrays (parallel readout, PIN diode arrays bump bonded to a CMOS readout). Additional new monolithic approaches including CMOS-SOI imagers are being developed at JPL and other institutions to address the need for separation of the detector and readout optimization while removing the cost and reliability concerns associated with hybridized approach using bump bonding. Such imager technologies offer the potential for high degree of integration to reduce the system mass and cost, low-power to reduce the thermal load, high-yield to lower procurement cost and highreliability to preserve mission life. Choice of the detector architecture will largely depend on the science requirements and instrument design. While existing CCD options likely have sufficient performance, CTE, power dissipation, 
integration and cost are primary reasons for the development of alternative imaging technologies such as CMOS. The CMOS FPAs will have to have performance comparable to CCDs. Areas of development include: i) devices offering wide spectral bandwidth (long wavelength response to $\lambda \geq 1 \mu \mathrm{m}$ and ii) photon counting (devices with sub-electron noise and/or photoconductive gain).

Regardless of readout schemes, to achieve the highest performance in quantum efficiency (QE), spectral range, fill factor, and dark current (surface-generated), silicon imagers must be back illuminated with proper processes such as JPL-invented delta doping technology.

\subsection{Packaging and Assembly}

Modular construction permits any single detector to be easily and independently removed and replaced without adversely affecting other detectors in the array. This modularity of the tiles in a large mosaic focal plane and the ability to replace or troubleshoot individual modules will affect the cost and reliability of the instrument. CCD, CMOS imagers, and hybrid arrays that could populate the FPA, can employ bump bonding between an imager in the array and its associated control/readout electronics for tight integration. Alternatively, flex cabling for this interface can be used to provide separation and minimize the electronics thermal load on the FPA.

Array tile alignment and systems packaging is critical for successful large focal plane arrays. The tile seam width directly affects science return and observational strategies. Completed detectors also have to be mated with a mechanical interface that enables the detector to meet the optical specifications of the instrument. Typical FPA planarity requirements at present are on the order of $\pm 10 \mu \mathrm{m}$ and the XY location of each detector will need to be controlled to a few tens of microns.

Mounting of the detector onto a stable interface is expected to accommodate and remove any departures from planarity inherent in free-standing processed silicon wafers. The mounted detector array has to meet the optical specifications at its operating temperature and it has to survive ground testing and orbital temperature cycling extending over the life of the mission. We believe that while metrology will be needed to assure that opto-mechanical specs have been met for the fully assembled focal plane, the mechanical tolerances required to meet the optical specifications are better addressed within the individual modules, during their design and fabrication, rather than at the focal plane during assembly.

Some of the above challenges have been faced in previous FPAs such as Kepler but there remains much room for improvement in areas such as power and tile seam size. In other missions such as Herschel-Planck and the Mars Science Laboratory, array packaging has been developed by JPL using different paradigms. Techniques involving sub-micron optical alignment, laser welding and hermetic packages have been developed and demonstrated over many $(>2000)$ extreme temperature (down to $\mathrm{mK}$ to $500^{\circ} \mathrm{C}$ ) cycles in these and developmental endeavors.

\subsection{Impact of Detector Fabrication Process Yield and Acceptance Criteria}

In an era of large FPAs, the required number of flight-rated detectors will be substantially larger than was needed for current missions. Using the SFC instrument concept as an example, 540 flight-rated detectors will be needed to successfully tile the two required focal planes. This is an order of magnitude larger than the Kepler mission. Complicating matters is the set of strict cosmetic and performance-based selection criteria that are commonly used today to select detectors for flight. It has been typical therefore to see many lot runs purchased to yield as few as 4 flight-rated detectors, resulting in a net yield of $<10 \%$. Clearly, without investment in new detector fabrication methodologies and infrastructure, these twin constraints of large detector quantities and low yields would result in extremely high costs and prohibitively long delivery schedules.

To address this issue, we need investment in hardware such as high-capacity Si-MBE systems that enable up to 8-inch wafer level processing of detectors, as well as batch processing of multiple wafers to the point where a lot run of wafers can be processed in record time. This kind of capability would dramatically increase the throughput and reproducibility over the equivalent die level processes. As 10's or 100's of detectors can be processed at the same time, wafer level processing alone results in almost an order of magnitude reduction in the time and labor costs required to fabricate scientific grade detectors. Wafer level processing also reduces variability between detectors because they are processed together. These tighter distributions in device quality enable one to systematically improve yield through process optimization. Thus, more high quality detectors can be produced in each run, and the time to complete a run is reduced.

In parallel with these process and infrastructure development investments, the impact of less restrictive flight device selection criteria should also be explored. For example, relaxing the selection criteria to include devices with more bad 
columns, hot pixels, and pixel-to-pixel variations in DQE that depart from typical specs of 3\% or less would clearly reduce mission costs. Such device imperfections could be dealt with by an expanded and more creative calibration program to minimize the risk to mission science goals. For example, a foveal construct can be employed where "perfect" devices would populate the most important central position(s) in the FPA. The lesser performing devices may then be kept for fringe locations. This could improve the yield and thereby reduce the cost while improving the observational capability of the instrument along with SyFT/PRODIGY paradigm. The large FPA era requires new paradigms for device fabrication and selection, and forward-thinking criteria to produce and down select devices for flight while keeping the mission costs and risk minimal.

\subsection{Testing}

While the construction of large numbers of detectors for large focal plane arrays is a challenging problem, the subsequent testing of those same numbers of detectors can potentially be overwhelming. The definition of a straightforward, quick and efficient testing program is critical to making the construction of such arrays even plausible. One can envision three levels of testing of individual focal planes: (1) detailed characterization of a small number of devices to determine optimize device operation and evaluate performance trade-offs; (2) screening of a very large number of devices; and (3) characterization of screened devices to bin them for flight, engineering, spares and rejects.

The development of automated cryogenic test systems (ACTS) will be essential for both quality control and throughput for these tests. In addition to addressing a list of detector performance issues, such test systems must also be configured to address new issues such as inter-operability between the detector and its control electronics and between these detector electronics and those off the focal plane. Such systems must also be capable of operating multiple detectors side by side to demonstrate that interference (crosstalk) is acceptable.

Experience strongly suggests the need to develop experimental testbeds to exercise the focal planes under simulated observation conditions to verify operational protocols, mitigate or calibrate undesirable characteristics and determine relevant selection criteria for engineering and flight qualified devices. The suggested effort is a new capability that is traditionally NOT part of detector performance testing.

\section{SCIENCE ENABLED BY THE STAR FORMATION CAMERA}

The SFC will address pivotal components in the 2007 NASA Science Plan: (2) Cosmic origins; and (3) Exoplanet exploration. The final design will provide $>100$ times greater imaging efficiency at 200-1100 nm than existed on previous missions. The mission has a well-defined Origins scientific program at its heart: a comprehensive, statistically significant survey of local, intermediate, and high-redshift sites and indicators of star formation, to investigate and understand the range of environments, feedback mechanisms, and other factors that most affect the outcome of the star and planet formation process and the path from the Big Bang to people. Stepping out from the nearest star-forming regions within our own Galaxy (Step 1), via the Magellanic Clouds (Step 2), to other nearby galaxies (Step 3), and on to the earliest cosmic epoch of galaxy assembly (Step 4), each step will build on the detailed knowledge gained at the previous step.

\subsection{Star and Planet Formation in the Milky Way ${ }^{5}$}

We will conduct a comprehensive imaging survey of Galactic star-forming regions, with the aim of tracking the evolution of circumstellar protoplanetary disks and other aspects of star formation in a wide variety of environments. This survey will combine multi-wavelength broad-band and key narrow-band imaging and will provide the measurements for color-color and color-magnitude diagram modeling of millions of young stars in a variety of evolutionary stages. This program will provide the basic data needed to understand star formation as a fundamental astrophysical process, will shed light on the apparent universality of the stellar IMF, and will be the cornerstone for interpreting measurements on star forming regions and global star formation properties in more distant galaxies.

\subsection{An Imaging Survey of the Magellanic Clouds ${ }^{6}$}

We will conduct a complete-area imaging survey of both Magellanic Clouds in 8 broad-band and 4 nebular emission-line filters that span the full $190-1075 \mathrm{~nm}$ wavelength range. We aim to (1) obtain a complete census of the stellar populations within the Clouds; (2) investigate feedback from massive stars, both in HII-regions environments and in the diffuse, warm ISM; (3) quantitatively parametrize the stellar clustering properties; and (4) determine how giant, starbursting HII-regions like 30 Doradus differ from more modest HII-regions within the Milky Way. In less than 1 year, 
SFC will be able to map both Magellanic Clouds in their entirety at $<0 . " 1-0 . " 15$ spatial resolution in 8 broad-band filters spanning the mid-UV $(\sim 225 \mathrm{~nm})$ through near-IR $\left(\mathrm{Y} ; \lambda_{\mathrm{c}}=1020 \mathrm{~nm}\right)$ to $\mathrm{m}_{\mathrm{AB}}>26 \mathrm{mag}$ and in 4 key narrow-band filters to $\sim 10^{-16} \mathrm{erg} \mathrm{cm}^{-2} \mathrm{~s}^{-1} \operatorname{arcsec}^{-2}$. We will image 21 HII-regions through 3 additional narrowband filters, to provide more detailed nebular diagnostics that are used to understand these star-formation environments in terms of what we learned at even smaller scales in our own Galaxy.

\subsection{The Hundred Galaxies Survey (HuGS) and Local-to-Virgo Survey of Nearby Galaxies (LoVeSoNG) ${ }^{7}$}

We will learn how galaxies work through studies of their stars, ISM, and immediate environments. This program consists of two complementary parts: HuGS will provide deep observations of a moderatesized sample of $\sim 100$ nearby galaxies using a set of broad- and narrow-band filters that span the 200-1100 nm wavelength range; LoVeSoNG medium-deep observations of a larger number $(\sim 500)$ galaxies through a subset of these filters. HuGS will analyze the resolved and unresolved stellar populations in 100 archetypical galaxies through color-magnitude and color-color diagram fitting and population synthesis modeling of multi-band colors and will yield physical properties such as spatially resolved star formation histories. The ISM in each galaxy will be analyzed using key narrow-band filters that distinguish photospheric from shock heating and provide information on the metallicity of the gas. LoVeSoNG will sample, and provide statistics for, the full parameter space of physical conditions and environments in which stars form and will place the deeper, detailed properties of HuGS in the broader context of the galaxy environments - their satellite systems and interface with the cosmic web. These programs rely on SFC's powerful combination of a large $\left(\sim 19^{\prime} \times 15^{\prime}\right)$ field of view, wavelength agility, sensitivity, and angular resolution. They will be the first comprehensive galaxy surveys at $0 . " 04-0 . " 13(200-1100 \mathrm{~nm})$ diffraction-limited resolution, providing a 21 st century digital standard for testing our understanding of the processes that affect the assembly and evolution of galaxies of different forms and mass.

\subsection{A Deep, Medium, and Wide Field survey of Cosmic Dawn, the start of Galaxy Assembly, and SMBH/AGN- growth at $\mathrm{z} 6-\mathbf{8}^{8}$}

We will conduct a tiered imaging survey with SFC using 8 filters spanning 200-1100nm that will cover $\sim 1 \mathrm{deg}^{2}$ to $\mathrm{AB} \sim<27 \mathrm{mag}$ (at $10 \sigma$ point source sensitivity; DEEP), $\sim 3 \mathrm{deg}^{2}$ to $\mathrm{AB} \sim<26 \mathrm{mag}$ (MEDIUM), and $\sim 9 \mathrm{deg}^{2}$ to $\mathrm{AB} \sim<25 \mathrm{mag}$ (WIDE). Each tier combines pan-chromatic data from at least two separate epochs. This survey is an essential complement to the kind of survey JWST will provide $\left(\sim<0.1 \mathrm{deg}^{2}\right.$ to $\mathrm{AB} \sim<31 \mathrm{mag}$ at $\left.\lambda>1100 \mathrm{~nm}\right)$. We aim to: (1) measure the faint-end of the luminosity function (LF) from $\mathrm{z} \sim 8$ to $5-$ SFC will map the onset of Pop II star formation, the dawn of (dwarf) galaxy formation and, hence, the objects that likely completed the reionization of the universe by $\mathrm{z} \sim 6$; (2) measure mass- and environment-dependent galaxy assembly from $\mathrm{z} \sim 5$ to 1 , combining up to 8-filter photometric redshift estimates with spatially resolved (on scales of a few kpc) analysis of stellar populations and structure for $\sim 6 \times 10^{5}$ galaxies; (3) trace the strongly epoch-dependent merger rate through early-stage mergers that were abundant at higher redshifts and constrain how 3 affected galaxy assembly; and (4) study in detail $\sim 10^{4}$ faint, variable objects-likely feeding weak AGN - over several $\mathrm{deg}^{2}$ and measure how growth of super-massive black holes and galaxy bulges kept pace through feedback processes.

\subsection{SFC study of the First Galaxies and Ly $\alpha$ Emitters ${ }^{8}$}

We will understand how galaxies formed from the perturbations in the primordial density field. By studying galaxies at $\mathrm{z}>6$ we can observe the original metal enrichment of the intergalactic medium (IGM) and the late stages of its reionization. A large survey is needed to build the statistics necessary to assess the strong spatial variations expected as a result of the galaxy formation and assembly process. The targets are very faint, so the observations need to be deep. The contribution of Lya emitters to the reionization of the IGM can be measured by studying the overlap phase, when the individual Strömgren spheres started to merge.

In addition, we intend to probe into the epoch of reionization and conduct a wide-field ( 1 deg $\left.{ }^{2}\right)$, narrow-band survey for Ly $\alpha$ emitting primordial galaxies at $6 \leq \mathrm{z} \leq 8$, well beyond current limits. JWST will not cover the wide areas required and none of the JDEM concepts provide the required narrow-band filters. The low near-infrared backgrounds of space combined with the small spatial sizes of Lya-emitting galaxies will make SFC the premier facility for studies of these important tracers of the assembly of the most clustered, massive galaxies. We aim to study their luminosity function to learn whether it changes dramatically at $\mathrm{z} \sim 6.5$, and to assess the cosmic importance of faint, low-luminosity systems relative to the rarer, bright, luminous systems at these redshifts. 


\section{REFERENCES}

[1] Kasdin, J., et al., "THEIA: Telescope for Habitable Exoplanets and Interstellar/Intergalactic Astronomy”, National Academy of Sciences, (2009), http://sites.nationalacademies.org/BPA/BPA 049855

[2] Scowen, P., et al., "The Star Formation Camera", National Academy of Sciences, (2009), http://sites.nationalacademies.org/BPA/BPA_049855

[3] Sembach, K., et al., "Technology Investments to Meet the Needs of Astronomy at Ultraviolet Wavelengths in the 21st Century", National Academy of Sciences, (2009), http://sites.nationalacademies.org/BPA/BPA 049522

[4] Scowen, P., et al., "Large Focal Plane Arrays for Future Missions", National Academy of Sciences, (2009), http://sites.nationalacademies.org/BPA/BPA 049522

[5] Scowen, P., et al., "Understanding Global Galactic Star Formation", National Academy of Sciences, (2009), http://sites.nationalacademies.org/BPA/BPA_050603

[6] Scowen, P., et al., "The Magellanic Clouds Survey: a Bridge to Nearby Galaxies", National Academy of Sciences, (2009), http://sites.nationalacademies.org/BPA/BPA 050603

[7] Jansen, R., et al., "A Systematic Study of the Stellar Populations and ISM in Galaxies out to the Virgo Cluster: nearfield cosmology within a representative slice of the local universe", National Academy of Sciences, (2009), http://sites.nationalacademies.org/BPA/BPA 050603

[8] Jansen, R., et al., "Galaxy Assembly and SMBH/AGN-growth from Cosmic Dawn to the End of Reionization", National Academy of Sciences, (2009), http://sites.nationalacademies.org/BPA/BPA_050603 